\title{
Prevalence of right ventricular dysfunction and prognostic significance in heart failure with preserved ejection fraction
}

\author{
Prathap Kanagala ${ }^{1,2,3}$ (D) Jayanth R. Arnold ${ }^{1} \cdot$ Anvesha Singh ${ }^{1} \cdot$ Jamal N. Khan ${ }^{1} \cdot$ Gaurav S. Gulsin ${ }^{1} \cdot$ Pankaj Gupta $^{1}$. \\ lain B. Squire ${ }^{1} \cdot$ Leong L. $\mathrm{Ng}^{1} \cdot$ Gerry P. McCann ${ }^{1}$
}

Received: 10 May 2020 / Accepted: 22 July 2020 / Published online: 31 July 2020

(c) The Author(s) 2020

\begin{abstract}
There is a paucity of data characterizing right ventricular performance in heart failure with preserved ejection fraction (HFpEF) using the gold standard of cardiovascular magnetic resonance imaging (CMR). We aimed to assess the proportion of right ventricular systolic dysfunction (RVD) in HFpEF and the relation to clinical outcomes. As part of a single-centre, prospective, observational study, 183 subjects (135 HFpEF, and 48 age- and sex-matched controls) underwent extensive characterization with CMR. transthoracic echocardiography, blood sampling and six-minute walk testing. Patients were followed for the composite endpoint of death or HF hospitalization. RVD (defined as right ventricular ejection fraction $<47 \%$ ) controls was present in 19\% of HFpEF. Patients with RVD presented more frequently with lower systolic blood pressure, atrial fibrillation, radiographic evidence of pulmonary congestion and raised cardiothoracic ratio and larger right ventricular volumes. During median follow-up of 1429 days, $47 \%(n=64)$ of HFpEF subjects experienced the composite endpoint of death $(n=22)$ or HF hospitalization $(n=42)$. RVD was associated with an increased risk of composite events (Log-Rank $\mathrm{p}=0.001$ ). In multivariable Cox regression analysis, RVD was an independent predictor of adverse outcomes (adjusted Hazard Ratio [HR] 3.946, 95\% CI 1.878-8.290, $\mathrm{p}=0.0001$ ) along with indexed extracellular volume (HR 1.742, CI 1.176-2.579, $\mathrm{p}=0.006$ ) and E/E' (HR 1.745, CI 1.230-2.477, p=0.002). RVD as assessed by CMR is prevalent in nearly one-fifth of HFpEF patients and is independently associated with death and/or hospitalization with HF.

The trial was registered retrospectively on ClinicalTrials.gov (Identifier: NCT03050593). The date of registration was February $06,2017$.
\end{abstract}

Keywords Heart failure with preserved ejection fraction · Right ventricular dysfunction · Prevalence $\cdot$ Prognosis · Cardiovascular magnetic resonance imaging

Electronic supplementary material The online version of this article (https://doi.org/10.1007/s10554-020-01953-y) contains supplementary material, which is available to authorized users.

Prathap Kanagala

pkk12@leicester.ac.uk

Jayanth R. Arnold

jra14@leicester.ac.uk

Anvesha Singh

as707@1eicester.ac.uk

Jamal N. Khan

mally777@ hotmail.com

Gaurav S. Gulsin

gg149@leicester.ac.uk

Pankaj Gupta

pankaj_gupta54@hotmail.com

Iain B. Squire

is11@leicester.ac.uk
Leong L. Ng

lln1@leicester.ac.uk

Gerry P. McCann

gpm12@leicester.ac.uk

1 Department of Cardiovascular Sciences, National Institute for Health Research (NIHR) Leicester Biomedical Research Centre, University of Leicester, Leicester, UK

2 Aintree University Hospital, Liverpool, UK

3 Department of Cardiovascular Sciences, Glenfield Hospital, Groby Road, Leicester LE3 9QP, UK 


\section{Introduction}

The importance of right ventricular (RV) function and its impact upon functional status [1] and outcomes [2] in heart failure with reduced ejection fraction (HFrEF) is well established. However, heart failure with preserved ejection fraction (HFpEF) currently accounts for approximately half of all cases of heart failure [3] and the role of right ventricular systolic dysfunction (RVD) in this setting is less well studied. To date, the majority of evidence for RVD is largely derived from echocardiographic data [4]. Moreover, the reported prevalence of RVD in HFpEF varies depending upon the choice of RV assessment tool and differing diagnostic thresholds (e.g. tricuspid annular plane systolic excursion [TAPSE], fractional area change [FAC], right ventricular ejection fraction [RVEF]) [5].

Cardiovascular magnetic resonance imaging (CMR) is the gold standard for RV volumetric and functional assessment, providing excellent accuracy and reproducibility $[6$, 7]. However, only 2 CMR studies [8, 9] have assessed $\mathrm{RV}$ function in in HFpEF, again with differing thresholds for RVD and both lacking reference control groups. All of the above observations were recognized in a position statement from the Heart Failure Association of the European Society of Cardiology, proposing further prospective outcome studies to identify clear cut-off values for RVD that are prognostically and clinically relevant [4]. In our prospective, observational study comprising both groups of HFpEF and age- and sex-matched healthy subjects, we aimed to assess the proportion of patients with RVD and explored the relation to clinical outcomes.

\section{Materials and methods}

\section{Study population}

HFpEF patients were recruited as part of the Developing Imaging And plasMa biOmarkers iN Describing HFpEF (DIAMOND-HFpEF) study: an observational, cohort study conducted at a single tertiary cardiac centre [10]. The National Research Ethics Service approved the study. All subjects provided written informed consent prior to participation. As detailed previously [11, 12] HFpEF inclusion criteria were: clinical or radiographic evidence of heart failure (HF), left ventricular (LV) ejection fraction (EF) $>50 \%$ on transthoracic echocardiography (TTE) and age $\geq 18$ years. Exclusion criteria were: known myocardial infarction (MI) in the preceding 6 months; suspected or confirmed cardiomyopathy or constrictive pericarditis; non-cardiovascular life expectancy $<6$ months; severe native valve disease; severe lung disease (or forced expiratory volume $\left[\mathrm{FEV}_{1}\right]<30 \%$ predicted or forced vital capacity $[\mathrm{FVC}]<50 \%$ predicted); estimated glomerular filtration rate $(\mathrm{eGFR})<30 \mathrm{ml} / \mathrm{min} / \mathrm{m}^{2}$ and standard contraindications to CMR.

A control group of 48 asymptomatic, age- and sexmatched subjects without known cardiac disease were also recruited. Since hypertension is highly prevalent in the general population without heart failure and is also strongly associated with incident HFpEF development, we included a subset of hypertensive controls $(n=22)$ in order to account for this potential confounder. All study participants underwent comprehensive clinical evaluation, blood sampling, TTE, six-minute walk testing (6MWT) and CMR during a solitary study visit.

\section{Blood sampling}

Blood was sampled for measurement of B-type natriuretic peptide (BNP; immunoassay, Siemens, Erlangen, Germany), haematocrit, haemoglobin and renal function (urea, creatinine).

\section{Transthoracic echocardiography}

As reported previously $[11,12]$ image acquisition and analysis was undertaken by 2 experienced, accredited sonographers using an iE 33 system with S5-1 transducer (Philips Medical Systems, Best, The Netherlands). Echocardiography was performed primarily to confirm preserved LVEF for study inclusion and $\mathrm{E} / \mathrm{E}^{\prime}$ was also calculated to assess LV filling pressure.

\section{Functional assessment of exercise capacity}

To provide an objective metric of exercise capacity, the 6MWT distance was measured in all subjects according to standardized protocols [13].

\section{Chest radiography}

The radiology reports of the most recent chest X-ray prior to the study visits were sourced from the hospital computerized reporting system [10]. The presence of pulmonary congestion and an enlarged cardiothoracic ratio were recorded. All reporting was done by Radiologists blinded to study participation and prior to subject enrolment.

\section{CMR protocol}

The CMR protocol used has been reported previously [11, $14,15]$. All scans were performed on a $3 \mathrm{~T}$ scanner (Siemens Skyra, Erlangen, Germany) with an 18-channel cardiac coil. 
In summary, the protocol included: conventional long- and short-axis cine images covering the LV and RV; pre- and post-contrast T1 mapping of basal, mid and apical LV slices; and late gadolinium enhancement (LGE) imaging. The total contrast dose administered was $0.15 \mathrm{mmol} / \mathrm{kg}$ of Gadovist (Bayer Healthcare, Berlin, Germany).

\section{CMR image analysis}

Cine images were analyzed using semi-automated cvi42 software (Circle Cardiovascular Imaging, Calgary, Canada) by a single experienced observer (PK), blinded to all clinical data. All volumetric and mass data were indexed to body surface area (BSA). Ventricular volumes (see Fig. 1), ejection fraction and LV mass (excluding papillary muscles) were calculated from the short-axis cine stack as previously described [10, 15]. The biplane method, excluding the appendage and pulmonary veins was used to measure maximal, minimal left atrial volumes (LAVmax, LAVmin) and derive left atrial ejection fraction (LAEF) [11]. A cutoff RVEF value of $<47 \%$ was used to define RVD based upon existing normative data from the published literature utilizing the same technique as in our study [16] as well as from our own healthy control data whereby the lower limit of RVEF was also 47\%. Qualitative LGE assessment was undertaken by 2 experienced operators to define the presence of MI as per standard criteria [15, 17]. In cases of disagreement, final adjudication was deferred to a third operator (GPM). As previously reported by our group with

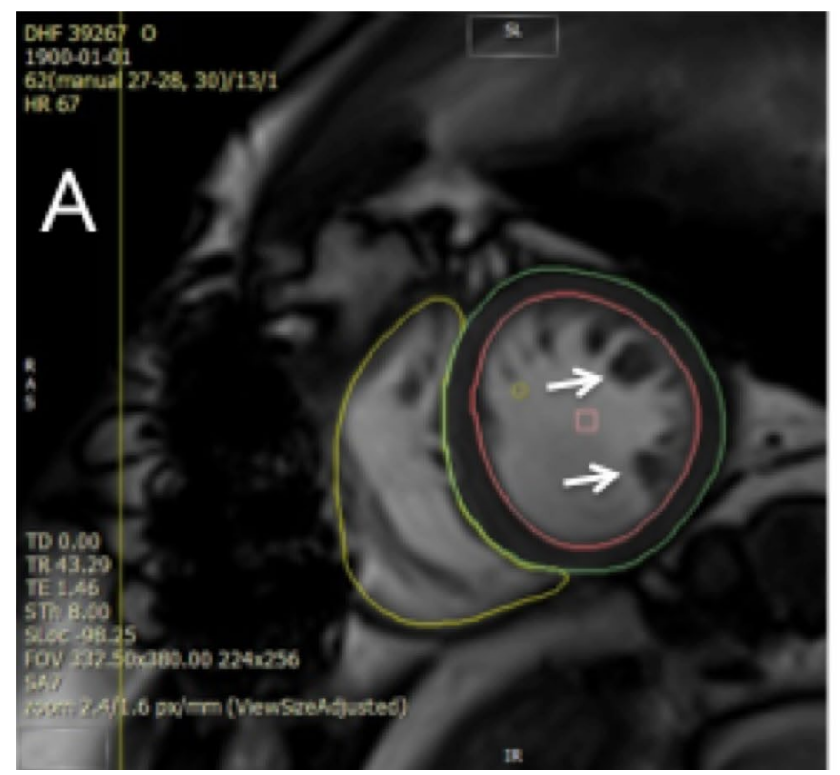

Fig. 1 Assessment of ventricular volumes, function and mass. Enddiastolic (a) and end-systolic (b) cine frames illustrating manually drawn contours of the left ventricular endocardium (pink), left ventricular epicardium (green) and right ventricular endocardium excellent reproducibility [18] and intra/inter-observer agreements [15], extracellular volume (ECV) and indexed ECV were quantified from mid short-axis LV slice T1 maps.

\section{Outcome data}

All participants were followed up for the primary endpoint, a composite of all-cause mortality or HF hospitalization (defined as a hospital admission for HF which required diuretic, inotropic or intravenous nitrate therapy). In patients experiencing multiple events, the time to first event was used as the censored outcome. Outcome data were obtained from Hospital databases. All patients had a minimum of 12 months follow-up, post study entry.

\section{Statistical analysis}

Statistical analyses were performed using SPSS V22. A p value of $<0.05$ was considered significant. Normality for continuous data was assessed using histograms, Q-Q plots and the Shapiro-Wilk test. Continuous data are presented as mean ( \pm standard deviation) or median $(25-75 \%$ interquartile range or range). Categorical data are presented as absolute numbers or percentages. Between group differences were compared using the $t$ test, Mann-Whitney U test and the Chi-square test as appropriate. BNP, creatinine, 6MWT distance and RVEF were $\log _{10}$ transformed before analysis.

Spearman's rank correlations were performed to check for important associations of other continuous variables

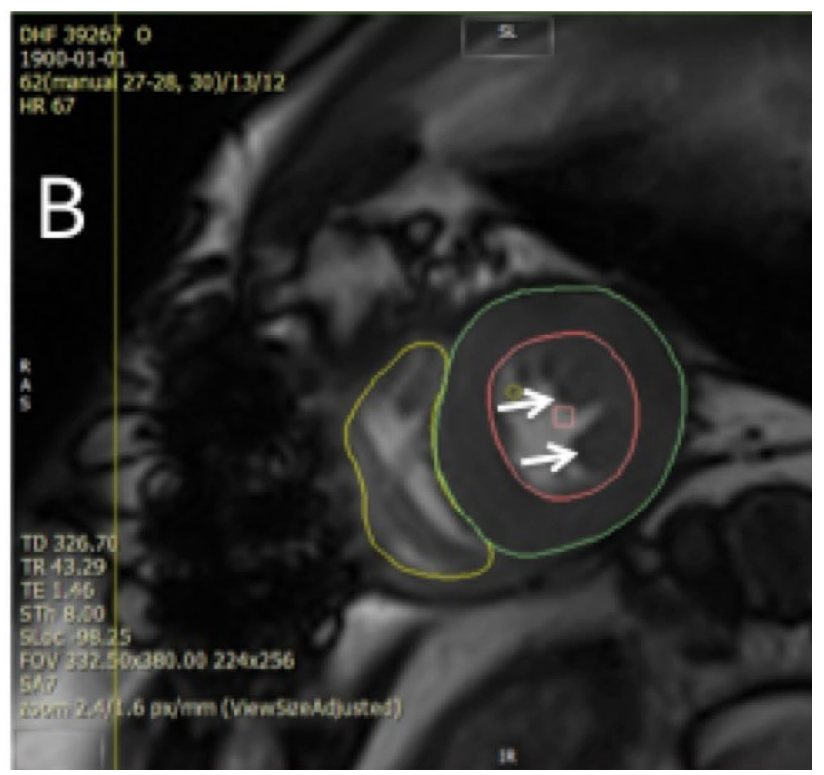

(yellow) for volumetric and mass analysis. (white arrows) papillary muscles and trabeculations were excluded from left ventricular mass calculations 
with RVEF. Intra-observer and inter-observer variability assessments for RV parameters were undertaken (by PK and JRA), a minimum of 4 weeks apart on 10 randomly selected patients.

Event rates were calculated from Kaplan-Meier survival analysis. Survival curve differences were assessed using the Log-Rank test. Univariable Cox regression modeling was initially performed to identify variables associated with outcomes. Only parameters associated with endpoints at $p<0.1$ were entered into subsequent multivariable analysis to identify independent predictors using both backwards and forwards stepwise elimination methods. In cases of collinearity, only variables with the highest coefficients or which have shown historically stronger prognostic importance based upon published literature were entered into multivariable analysis. To further minimize over-fitting, Cox regression models were limited to one parameter per approximately 10 composite events. Four separate, clinically relevant models were created including a final model comprising the strongest predictors. Continuous variables were further Z-standardized to enable comparison of hazard ratios (HR) based upon one standard deviation increase in the predictor variable. The accuracy of the final independent Cox model to predict events was then tested by receiver operator characteristics (ROC) analysis.

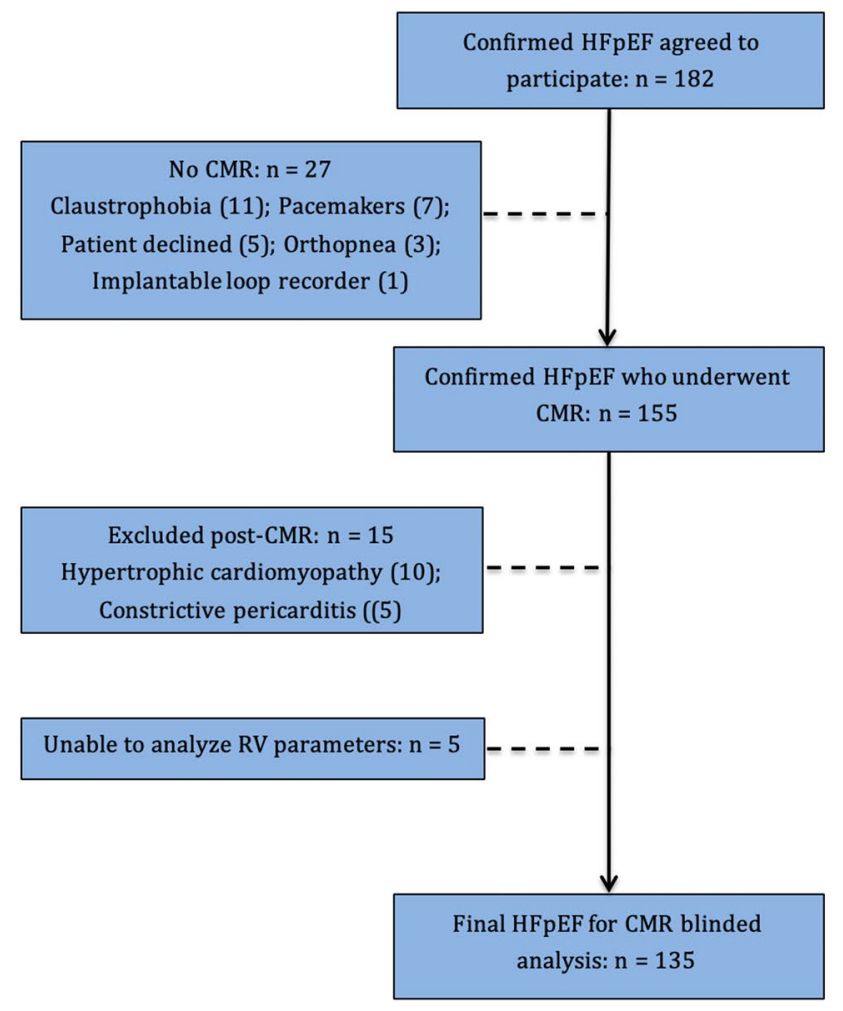

\section{Results}

Two hundred and thirty two subjects were enrolled (HFpEF $n=182$, controls $n=50$ ), of whom 49 were excluded (see Fig. 2) from further analysis. Of these, RV assessment could not be performed in 5 patients due to degraded image quality. Our final cohort who underwent RV analysis comprised a total of 183 participants (HFpEF $n=135$, controls $n=48$ ). As previously reported, iECV calculation was not possible (HFpEF $n=43$, controls $n=4$ ) in a small subset due to the unavailability of the sequences for T1 mapping [15]. Both intra-observer and inter-observer variability were excellent for RV parameters [10]. All subjects were recruited over a period of 26 months. The final participant was enrolled in April 2015. Follow-up was until January 2019. Baseline demographic features of patients with $\mathrm{HFpEF}$ and control subjects are shown in Table1; imaging data are in Table 2.

\section{Comparison of HFpEF and controls}

Overall, HFpEF and healthy controls were well matched for age ( $73 \pm 9$ years) and sex. Approximately two-thirds of HFpEF patients had experienced prior hospitalization for HF or had radiographic evidence of pulmonary congestion.

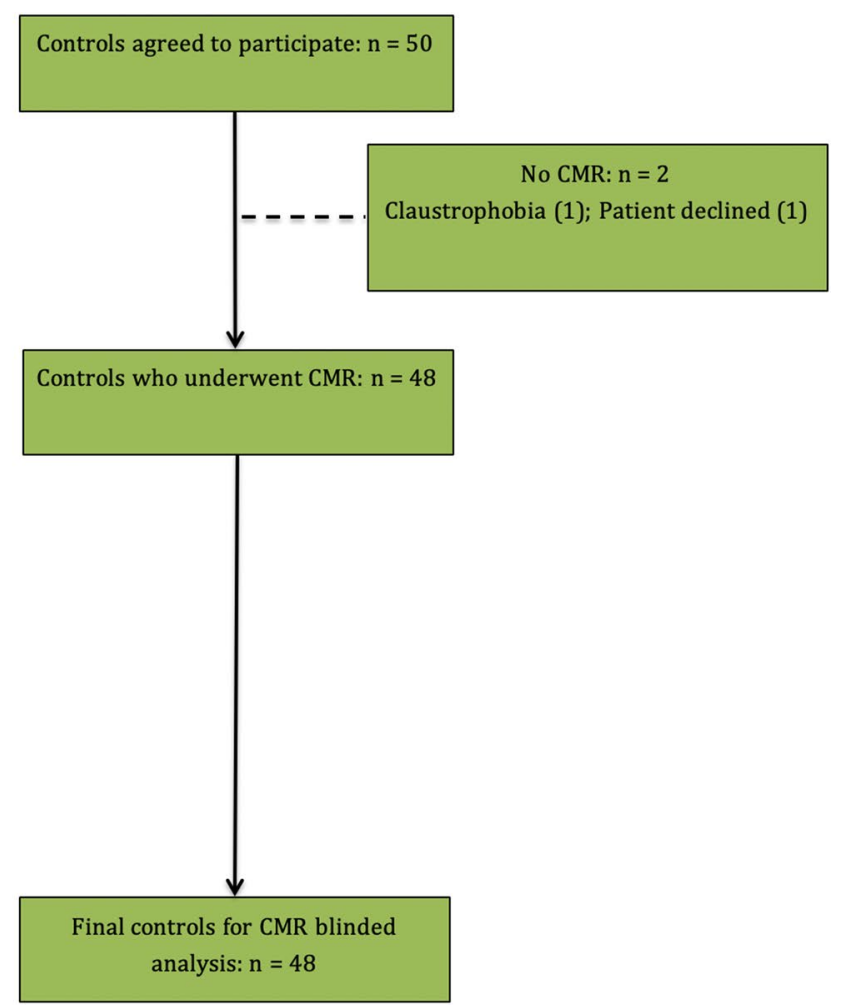

Fig. 2 Study recruitment overview. Flow chart illustrating recruitment and reasons for exclusion. CMR cardiovascular magnetic resonance imaging, $H F p E F$ heart failure with preserved ejection fraction, $R V$ right ventricle 
Table 1 Baseline clinical characteristics

$\begin{array}{llllll}\begin{array}{l}\text { Controls } \\ \mathrm{n}=48\end{array} & \text { HFpEF } & \text { p value } & \text { HFpEF } & \text { HFpEF with RVD } \\ & & \begin{array}{l}\text { No RVD } \\ n=135\end{array} & n=25 & \\ & & & \\ \end{array}$

\begin{tabular}{|c|c|c|c|c|c|c|}
\hline \multicolumn{7}{|l|}{ Demographics } \\
\hline Age (years) & $73 \pm 5$ & $72 \pm 9$ & 0.521 & $72 \pm 9$ & $75 \pm 11$ & 0.183 \\
\hline Male $(\%)$ & $24(50)$ & $66(49)$ & 0.895 & $51(46)$ & $15(60)$ & 0.218 \\
\hline \multicolumn{7}{|l|}{ Clinical findings } \\
\hline Heart rate (b.p.m.) & $68 \pm 10$ & $70 \pm 14$ & 0.195 & $70 \pm 14$ & $70 \pm 14$ & 0.991 \\
\hline Systolic BP (mmHg) & $151 \pm 24$ & $145 \pm 25$ & 0.193 & $147 \pm 25$ & $136 \pm 26$ & 0.042 \\
\hline Diastolic BP (mmHg) & $79 \pm 10$ & $74 \pm 12$ & 0.016 & $74 \pm 12$ & $74 \pm 14$ & 0.924 \\
\hline Body mass index $\left(\mathrm{kg} / \mathrm{m}^{2}\right)$ & $25 \pm 3$ & $34 \pm 7$ & $<0.0001$ & $34 \pm 7$ & $33 \pm 7$ & 0.623 \\
\hline Sinus rhythm (\%) & $48(100)$ & $94(70)$ & $<0.0001$ & $82(75)$ & $12(48)$ & 0.009 \\
\hline Atrial fibrillation (\%) & $0(0)$ & $41(30)$ & $<0.0001$ & $28(25)$ & $13(52)$ & 0.009 \\
\hline \multicolumn{7}{|l|}{ Medical history } \\
\hline Prior hospitalization with heart failure & NA & $89(66)$ & NA & $67(61)$ & $22(88)$ & 0.010 \\
\hline Diabetes $(\%)$ & $0(0)$ & $67(50)$ & $<0.0001$ & $54(49)$ & $13(52)$ & 0.793 \\
\hline Hypertension (\%) & $22(46)$ & $122(90)$ & $<0.0001$ & $97(88)$ & $25(100)$ & 0.071 \\
\hline Angina $(\%)$ & $0(0)$ & $22(16)$ & 0.003 & $18(16)$ & $4(16)$ & 0.965 \\
\hline Known myocardial infarction $(\%)$ & $0(0)$ & $15(11)$ & 0.016 & $13(12)$ & $2(8)$ & 0.583 \\
\hline Known coronary artery disease $(\%)$ & $0(0)$ & $30(22)$ & $<0.0001$ & $24(22)$ & $6(24)$ & 0.813 \\
\hline Asthma or COPD (\%) & $3(6)$ & $21(16)$ & 0.101 & $16(15)$ & $5(20)$ & 0.497 \\
\hline Smoking (\%) & $17(35)$ & $71(53)$ & 0.041 & $59(54)$ & $12(48)$ & 0.610 \\
\hline Hypercholesterolameia (\%) & $18(38)$ & $68(50)$ & 0.125 & $56(51)$ & $12(48)$ & 0.793 \\
\hline Peripheral vascular disease $(\%)$ & $0(0)$ & $3(2)$ & 0.298 & $2(2)$ & $1(4)$ & 0.504 \\
\hline TIA or CVA $(\%)$ & $1(2)$ & $19(14)$ & 0.005 & $16(15)$ & $3(1)$ & 0.770 \\
\hline \multicolumn{7}{|l|}{ Medication } \\
\hline Betablocker (\%) & $2(4)$ & $93(69)$ & $<0.0001$ & $72(65)$ & $21(84)$ & 0.071 \\
\hline ACEi or ARB $(\%)$ & $10(21)$ & $116(86)$ & $<0.0001$ & $95(86)$ & $21(84)$ & 0.759 \\
\hline Aldosterone antagonist (\%) & $0(0)$ & $42(31)$ & $<0.0001$ & $32(29)$ & $10(40)$ & 0.288 \\
\hline Loop diuretic $(\%)$ & $0(0)$ & $108(80)$ & $<0.0001$ & $86(78)$ & $22(88)$ & 0.268 \\
\hline \multicolumn{7}{|l|}{ Functional status } \\
\hline NYHA I/II (\%) & NA & $95(70)$ & NA & $80(75)$ & $15(60)$ & 0.208 \\
\hline NYHA III/IV (\%) & NA & $40(30)$ & NA & $30(27)$ & $10(40)$ & 0.208 \\
\hline Six minute walk distance (m) & $380(350-440)$ & $185(120-250)$ & $<0.0001$ & $190(130-250)$ & $180(100-260)$ & 0.633 \\
\hline \multicolumn{7}{|l|}{ Bloods } \\
\hline Sodium $(\mathrm{mmol} / \mathrm{l})$ & $140.4 \pm 1.7$ & $139.3 \pm 3.5$ & 0.007 & $139.2 \pm 3.3$ & $139.6 \pm 4.2$ & 0.661 \\
\hline Urea $(\mathrm{mmol} / \mathrm{l})$ & $6.1 \pm 1.5$ & $8.3 \pm 3.4$ & $<0.0001$ & $8.4 \pm 3.4$ & $8.0 \pm 3.5$ & 0.613 \\
\hline Creatinine (mmol/l, IQR) & $70.5(56.3-84.5)$ & $88(73-113)$ & $<0.0001$ & $90(73-116)$ & $84(70-108)$ & 0.283 \\
\hline Haemoglobin (g/l) & $140 \pm 15$ & $129 \pm 22$ & $<0.0001$ & $129 \pm 22$ & $127 \pm 21$ & 0.658 \\
\hline $\mathrm{BNP}$ (ng/l, IQR) & $33(24-44)$ & $136(65-256)$ & $<0.0001$ & $134(54-269)$ & $170(84-245)$ & 0.428 \\
\hline
\end{tabular}

Values are mean $\pm \mathrm{SD}$ or $\mathrm{n}(\%)$ or median (interquartile range). The $\mathrm{p}$ values are for the t-test, Mann-Whitney U test or Chi-square test as appropriate

$A C E i$ angiotensin converting enzyme inhibitor, $A R B$ angiotensin II receptor blocker, $B N P$ B-type natriuretic peptide, $C M R$ cardiovascular magnetic resonance imaging, COPD chronic obstructive pulmonary disease, $C V A$ cerebrovascular accident, $N A$ not applicable or not assessed, $N Y H A$ New York Heart association, TIA transient ischaemic attack

HFpEF was frequently associated with co-morbidities including obesity, diabetes, hypertension, atrial fibrillation (AF), renal dysfunction and anaemia. Significant minorities of HFpEF also had known ischaemic heart disease (22\%) and lung disease (16\%). Furthermore, HFpEF patients had worse exercise capacity (shorter 6MWT distance) and nearly a third were classed as NYHA III/IV.

Metrics of diastolic dysfunction (BNP, E/E'), and LV mass) were higher in HFpEF. Compared to control subjects $(58 \pm 5 \%)$, LVEF was marginally lower in HFpEF $(56 \pm 5 \%$, 
Table 2 Baseline imaging characteristics

\begin{tabular}{|c|c|c|c|c|c|c|}
\hline & $\begin{array}{l}\text { Controls } \\
\mathrm{n}=48\end{array}$ & $\begin{array}{l}\mathrm{HFpEF} \\
\mathrm{n}=135\end{array}$ & $\mathrm{p}$ value & $\begin{array}{l}\text { HFpEF } \\
\text { No RVD } \\
n=110\end{array}$ & $\begin{array}{l}\text { HFpEF with RVD } \\
\mathrm{n}=25\end{array}$ & $\mathrm{p}$ value \\
\hline \multicolumn{7}{|l|}{ Previous chest radiography } \\
\hline Pulmonary congestion (\%) & NA & $93(69)$ & NA & $71(65)$ & $22(88)$ & 0.025 \\
\hline Raised CTR (\%) & NA & $98(73)$ & NA & $75(68)$ & $23(92)$ & 0.018 \\
\hline Pleural effusion $(\%)$ & NA & $48(36)$ & NA & $36(33)$ & $12(48)$ & 0.159 \\
\hline \multicolumn{7}{|l|}{ Echo } \\
\hline $\mathrm{E} / \mathrm{E}^{\prime}$ & $9 \pm 3$ & $13 \pm 5$ & $<0.0001$ & $13 \pm 5$ & $13 \pm 6$ & 0.723 \\
\hline \multicolumn{7}{|l|}{ CMR } \\
\hline $\operatorname{LVEF}(\%)$ & $58 \pm 5$ & $56 \pm 5$ & 0.019 & $56 \pm 5$ & $55 \pm 6$ & 0.449 \\
\hline $\operatorname{LVEDVI}\left(\mathrm{ml} / \mathrm{m}^{2}\right)$ & $81 \pm 14$ & $79 \pm 18$ & 0.409 & $79 \pm 19$ & $77 \pm 16$ & 0.493 \\
\hline $\operatorname{LVMI}\left(\mathrm{g} / \mathrm{m}^{2}\right)$ & $46 \pm 9$ & $52 \pm 15$ & $<0.0001$ & $52 \pm 16$ & $52 \pm 10$ & 0.886 \\
\hline LV mass/LV volume & $0.57 \pm 0.09$ & $0.68 \pm 0.16$ & $<0.0001$ & $0.67 \pm 0.16$ & $0.70 \pm 0.15$ & 0.447 \\
\hline RVEF (\%), median, range & $55(47-70)$ & $54(27-74)$ & 0.090 & $56(47-74)$ & $44(27-46)$ & $<0.0001$ \\
\hline $\operatorname{RVEDVI}\left(\mathrm{ml} / \mathrm{m}^{2}\right)$ & $83 \pm 15$ & $80 \pm 19$ & 0.307 & $76 \pm 16$ & $98 \pm 20$ & $<0.0001$ \\
\hline $\operatorname{RVESVI}\left(\mathrm{ml} / \mathrm{m}^{2}\right)$ & $37 \pm 9$ & $37 \pm 14$ & 0.849 & $33 \pm 10$ & $57 \pm 15$ & $<0.0001$ \\
\hline \multicolumn{7}{|l|}{ Overall } \\
\hline LAEF $(\%)$ & $51 \pm 11$ & $32 \pm 16$ & $<0.0001$ & $35 \pm 15$ & $22 \pm 12$ & $<0.0001$ \\
\hline LAVImax $\left(\mathrm{ml} / \mathrm{m}^{2}\right)$ & $35 \pm 12$ & $53 \pm 25$ & $<0.0001$ & $51 \pm 23$ & $62 \pm 31$ & 0.054 \\
\hline $\operatorname{LAVImin}\left(\mathrm{ml} / \mathrm{m}^{2}\right)$ & $17 \pm 8$ & $38 \pm 25$ & $<0.0001$ & $36 \pm 23$ & $49 \pm 31$ & 0.017 \\
\hline \multicolumn{7}{|l|}{ Sinus rhythm } \\
\hline $\operatorname{LAEF}(\%)$ & $51 \pm 11$ & $41 \pm 12$ & $<0.0001$ & $42 \pm 11$ & $32 \pm 10$ & 0.006 \\
\hline $\operatorname{LAVImax}\left(\mathrm{ml} / \mathrm{m}^{2}\right)$ & $35 \pm 12$ & $43 \pm 16$ & 0.003 & $43 \pm 16$ & $44 \pm 17$ & 0.854 \\
\hline $\operatorname{LAVImin}\left(\mathrm{ml} / \mathrm{m}^{2}\right)$ & $17 \pm 8$ & $26 \pm 13$ & $<0.0001$ & $25 \pm 13$ & $30 \pm 15$ & 0.243 \\
\hline Presence of MI on LGE (\%) & $0(0)$ & $23(17)$ & 0.002 & $17(15)$ & $6(24)$ & 0.305 \\
\hline $\mathrm{iECV}\left(\mathrm{ml} / \mathrm{m}^{2}\right)$ & $10.9 \pm 2.8$ & $13.7 \pm 4.4$ & $<0.0001$ & $13.5 \pm 4.5$ & $14.7 \pm 3.7$ & 0.276 \\
\hline
\end{tabular}

Values are mean $\pm \mathrm{SD}$ or $\mathrm{n}(\%)$. The $\mathrm{p}$ values are for the t-test, Mann-Whitney $\mathrm{U}$ test or Chi-square test as appropriate

$C T R$ cardiothoracic ratio, $i E C V$ indexed extracellular volume, $L A E F$ left atrial ejection fraction, $L A V I$ left atrial volume indexed to body surface area (maximal/minimal), $L G E$ late gadolinium enhancement, $L V M I$ left ventricular end-diastolic mass indexed to body surface area, $L V E D V I$ left ventricular end-diastolic volume indexed to body surface area, $M I$ myocardial infarction, $N A$ not applicable or not assessed, $R V E F$ right ventricular ejection fraction, RVEDVI right ventricular end-diastolic volume indexed to body surface area, RVESVI right ventricular end-systolic volume indexed to body surface area

$\mathrm{p}=0.019)$, albeit preserved overall. More concentric remodeling (higher LV mass/volume) and diffuse fibrosis (iECV) was also evident in HFpEF. LA remodeling (higher LAVImax, LAVImin) and dysfunction (lower LAEF) was highly prevalent in HFpEF, irrespective of cardiac rhythm. RVEF in the control group had a narrow range (median 55, 47-70) in contrast to HFpEF (median 54, 27-73), but the difference was not statistically significant $(p=0.090)$. No significant differences in RV volumes between HFpEF and controls were noted.

\section{Comparison of HFpEF with and without RVD}

RVD (was present in nearly one-fifth $(19 \%, n=25)$ of patients with HFpEF. Compared to patients without RVD, those with RVD presented more frequently with lower systolic blood pressure, AF, radiographic evidence of pulmonary congestion and elevated cardiothoracic ratio. RVD was associated with larger right ventricular and LA (LAVImin) volumes, and lower LAEF (irrespective of AF or sinus rhythm). Furthermore, prior hospitalization with decompensated HF was also more prevalent in this subgroup. There were no statistically significant differences between groups in terms of medical history, biochemical profiles and prescribed cardiac pharmacotherapies. While measures of functional status were worse in the RVD group i.e. greater proportion of NYHA III/IV and shorter 6 MWT distance walked, these differences did not reach statistical significance.

\section{Correlates of RVEF}

Statistically significant correlations of RVEF with other variables are shown in Table 3. Positive correlations were 
Table 3 Significant associations of RVEF with other continuous variables

\begin{tabular}{lcc}
\hline & $\begin{array}{l}\text { Correlation coefficients } \\
\text { (Spearman's) }\end{array}$ & p value \\
\hline Systolic BP $(\mathrm{mmHg})$ & 0.321 & $<0.0001$ \\
LVEDVI $\left(\mathrm{ml} / \mathrm{m}^{2}\right)$ & 0.174 & 0.044 \\
LVEF $(\%)$ & 0.219 & 0.011 \\
RVEDVI $\left(\mathrm{ml} / \mathrm{m}^{2}\right)$ & -0.392 & $<0.0001$ \\
RVESVI $\left(\mathrm{ml} / \mathrm{m}^{2}\right)$ & -0.788 & $<0.0001$ \\
LAVImax $\left(\mathrm{ml} / \mathrm{m}^{2}\right)$ & -0.175 & 0.043 \\
LAVImin $\left(\mathrm{ml} / \mathrm{m}^{2}\right)$ & -0.272 & 0.001 \\
LAEF $(\%)$ & 0.441 & $<0.0001$ \\
\hline
\end{tabular}

Abbreviations are as per Tables 1 and 2

observed with LVEF and LAEF. Inverse relationships were seen for RVEF with RV, LV and LA volumes. The strongest correlations were with RV volumes (right ventricular endsystolic volume indexed [RVESVI] $r=-0.788$, right ventricular end-diastolic volume indexed [RVEDVI $r=-0.392$ ] and LAEF $\mathrm{r}=0.441$ ).

\section{RVD and outcomes}

During median follow-up of 1429 days (1153-1654), 47\% of HFpEF subjects $(\mathrm{n}=64)$ experienced the composite endpoint of death $(n=22)$ or hospitalization with $\operatorname{HF}(n=42)$. There were no events in the control group.

Kaplan-Meier survival curves stratified according to the presence or absence of RVD in HFpEF are shown in Fig. 3. HFpEF patients with RVD had significantly higher event rates (Log-Rank $p=0.001)$. Furthermore, in those HFpEF patients with RVD, when stratified into tertiles

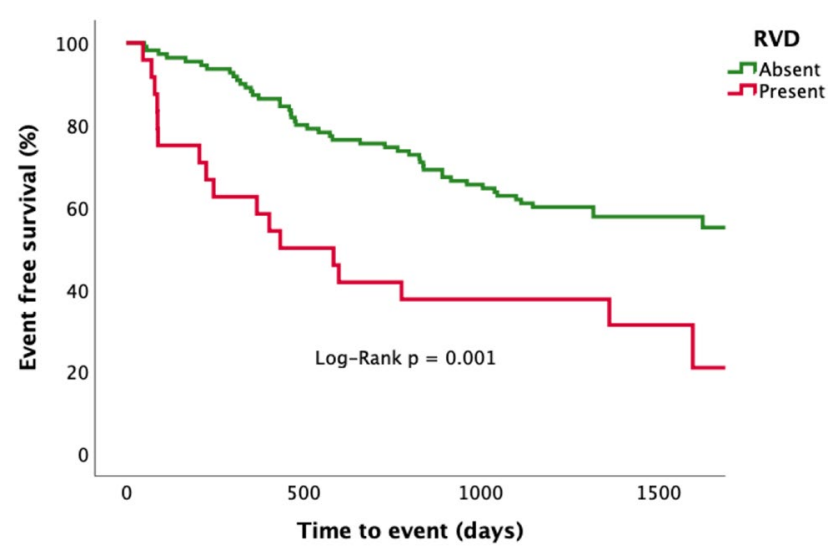

Fig. 3 Survival analysis stratified according to the presence or absence of right ventricular dysfunction. Kaplan-Meier analysis for the composite endpoint of death and/or hospitalization with heart failure; $R V D$ right ventricular dysfunction on the basis of RVEF (see online Resource Supplementary Fig. 1), the lower RVEF groups were associated with increasing risk of HF hospitalizations, albeit statistical significance was not reached ( $\log -\operatorname{Rank} p=0.170)$. On univariable Cox regression analysis (Table 4), nineteen parameters were associated with adverse outcomes: age, diastolic blood pressure, prior HF hospitalization, NYHA III/IV, Log 6MWT distance, Log creatinine, haemoglobin, Log BNP, E/E', left ventricular mass indexed (LVMI), LAVImax, LAEF, presence of MI on LGE, ECV indexed ECV, RVEDVI, RVESVI, Log RVEF and RVD. Of these, 4 parameters were excluded from multivariable analysis. During multivariable analysis (see Table 5), RVD remained significantly associated with outcomes in 3 separate models incorporating either: clinical, biochemical or imaging metrics. In a final model comprising the strongest parameters overall, RVD remained an independent predictor of outcomes (adjusted Hazard Ratio [HR] 3.946, 95\% CI 1.878-8.290, $\mathrm{p}=0.0001)$ along with indexed ECV (HR 1.742, CI 1.176-2.579, $\mathrm{p}=0.006$ ) and echocardiographic E/E' (HR 1.745, CI 1.230-2.477, $\mathrm{p}=0.002)$. The final Cox model incorporating these 3

Table 4 Unadjusted predictors for the composite endpoint of death and/or hospitalization with heart failure

\begin{tabular}{lll}
\hline Unadjusted predictors of outcome & & \\
\hline & Hazard ratio $(95 \% \mathrm{CI})$ & $\mathrm{p}$ value \\
\hline Clinical & & \\
Age (years) & $1.406(1.068-1.851)$ & 0.015 \\
Average diastolic BP (mmHg) & $0.660(0.505-0.863)$ & 0.002 \\
Prior HF hospitalization & $3.332(1.735-6.399)$ & 0.0001 \\
NYHA III/IV & $1.747(1.054-2.894)$ & 0.030 \\
Log 6MWT distance $(\mathrm{m})$ & $0.739(0.580-0.941)$ & 0.014 \\
Clinical blood samples & & \\
Log creatinine $(\mu \mathrm{mol} / \mathrm{l})$ & $1.281(1.021-1.607)$ & 0.032 \\
Haemoglobin $(\mathrm{g} / \mathrm{l})$ & $0.711(0.550-0.920)$ & 0.009 \\
Log BNP $(\mathrm{ng} / \mathrm{l})$ & $1.437(1.093-1.889)$ & 0.009 \\
Imaging & & \\
LV mass index $\left(\mathrm{g} / \mathrm{m}^{2}\right)$ & $1.284(1.028-1.605)$ & 0.028 \\
LAVImax $\left(\mathrm{ml} / \mathrm{m}^{2}\right)$ & $1.310(1.044-1.643)$ & 0.020 \\
Biplane LAEF $(\%)$ & $0.737(0.578-0.938)$ & 0.013 \\
LGE MI $(\%)$ & $1.745(0.963-3.159)$ & 0.066 \\
RVEDVI $\left(\mathrm{ml} / \mathrm{m}^{2}\right)^{\mathrm{a}}$ & $1.292(1.001-1.668)$ & 0.049 \\
RVESVI $\left(\mathrm{ml} / \mathrm{m}^{2}\right)^{\mathrm{a}}$ & $1.305(1.035-1.645)$ & 0.024 \\
Log RVEF $(\%)^{\mathrm{a}}$ & $0.819(0.650-1.030)$ & 0.088 \\
RVD & $2.533(1.452-4.419)$ & 0.001 \\
ECV $(\%)^{\mathrm{a}}$ & $1.578(1.144-2.178)$ & 0.005 \\
iECV $\left(\mathrm{ml} / \mathrm{m}^{2}\right)$ & $1.546(1.128-2.119)$ & 0.007 \\
E/E' & $1.420(1.118-1.804)$ & 0.004 \\
\hline
\end{tabular}

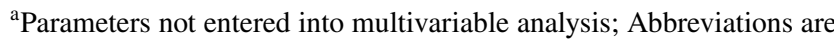
as per Tables 1 and 2 
Table 5 Multiple Cox regression models inclusive of RVD for the composite endpoint of death and/or hospitalization with heart failure

\begin{tabular}{|c|c|c|}
\hline \multicolumn{3}{|l|}{ Adjusted predictors of outcome } \\
\hline & Hazard ratio $(95 \% \mathrm{CI})$ & $\mathrm{p}$ value \\
\hline \multicolumn{3}{|l|}{ Clinical (Model 1) } \\
\hline Age (years) & $1.247(0.925-1.679)$ & 0.147 \\
\hline Average Diastolic BP (mmHg) & $0.735(0.572-0.944)$ & 0.016 \\
\hline Prior HF hospitalization & $2.671(1.360-5.245)$ & 0.004 \\
\hline NYHA III/IV & $0.869(0.391-1.929)$ & 0.729 \\
\hline Log 6MWT distance (m) & $0.883(0.665-1.173)$ & 0.392 \\
\hline$+\mathrm{RVD}$ & $1.873(1.054-3.327)$ & 0.032 \\
\hline \multicolumn{3}{|l|}{ Clinical blood samples (model 2) } \\
\hline Log Creatinine $(\mu \mathrm{mol} / \mathrm{l})$ & $1.296(1.034-1.624)$ & 0.025 \\
\hline Haemoglobin (g/l) & $0.764(0.591-0.987)$ & 0.040 \\
\hline $\log$ BNP (ng/l) & $1.228(0.925-1.630)$ & 0.155 \\
\hline$+\mathrm{RVD}$ & $2.495(1.419-4.384)$ & 0.001 \\
\hline \multicolumn{3}{|l|}{ Imaging (model 3) } \\
\hline LV mass index $\left(\mathrm{g} / \mathrm{m}^{2}\right)$ & $0.848(0.360-2.000)$ & 0.707 \\
\hline $\operatorname{LAVImax}\left(\mathrm{ml} / \mathrm{m}^{2}\right)$ & $0.742(0.414-1.332)$ & 0.318 \\
\hline Biplane LAEF (\%) & $0.827(0.575-1.189)$ & 0.306 \\
\hline LGE MI (\%) & $1.374(0.526-3.590)$ & 0.516 \\
\hline $\operatorname{iECV}\left(\mathrm{ml} / \mathrm{m}^{2}\right)$ & $1.742(1.176-2.579)$ & 0.006 \\
\hline $\mathrm{E} / \mathrm{E}^{\prime}$ & $1.745(1.230-2.477)$ & 0.002 \\
\hline$+\mathrm{RVD}$ & $3.946(1.878-8.290)$ & 0.0001 \\
\hline \multicolumn{3}{|c|}{ Strongest markers combined (model 4) } \\
\hline Average diastolic BP (mmHg) & $1.306(0.890-1.916)$ & 0.172 \\
\hline Prior HF hospitalization & $2.094(0.875-5.011)$ & 0.097 \\
\hline Log creatinine $(\mu \mathrm{mol} / \mathrm{l})$ & $1.343(0.929-1.941)$ & 0.116 \\
\hline Haemoglobin (g/l) & $0.983(0.634-1.525)$ & 0.940 \\
\hline $\mathrm{iECV}\left(\mathrm{ml} / \mathrm{m}^{2}\right)$ & $1.742(1.176-2.579)$ & 0.006 \\
\hline $\mathrm{E} / \mathrm{E}^{\prime}$ & $1.745(1.230-2.477)$ & 0.002 \\
\hline$+\mathrm{RVD}$ & $3.946(1.878-8.290)$ & 0.0001 \\
\hline
\end{tabular}

Abbreviations are as per Tables 1 and 2

independent variables to predict outcome yielded an area under the ROC curve of $0.732, \mathrm{p}<0.0001$.

\section{Discussion}

This is the first prospective study to analyze RV systolic performance and remodeling with CMR in both age- and sex-matched HFpEF and control groups. The principal findings in our study are that in HFpEF: (1) RVD is present in a significant minority; (2) RVEF is associated with $\mathrm{RV} / \mathrm{LV} / \mathrm{LA}$ volumes and LA function; and (3) RVD is independently associated with the risk of death or rehospitalization with HF.

\section{Prevalence of RVD}

To date, the reportedly wide range of prevalence of RVD in HFpEF of 4 to $44 \%$ has been derived almost exclusively from echocardiographic data [5]. Factors implicated in this variation in prevalence include the differing populations studied (community based, registry data, clinical trials) and variable definitions of both HFpEF (LVEF $\geq 45 \%$ and LVEF $>50 \%$ ) and RVD $[4,5]$. Besides, the complex geometry of the RV renders it a difficult chamber to assess with traditional 2D echocardiography, especially in the context of HFpEF when imaging may be more challenging due to co-morbidites such as lung disease, obesity and AF [4].

CMR is the established gold standard for RV assessment $[6,7]$. To date, only one prior CMR study [8] has reported prevalence (19\%) of RVD in HFpEF, using a RVEF cut-off of $<45 \%$, primarily based upon ARVC guidelines [19]. In contrast, we observed a similar prevalence using a slightly higher RVEF cut-off of RVEF $<47 \%$ based on our own internal reference controls, a particular strength of our study.

\section{Significance of RVD in HFpEF, causes and possible mechanisms implicated in outcomes}

In HFrEF, the presence of RVD portends poorer functional status, exercise capacity [1, 20] and prognosis [2]. However, a similar association of RVD with outcomes in HFpEF has not been observed consistently. In echocardiographic studies of community [21] and hospital based HFpEF subjects referred for invasive right heart catheterization [22], RVD was independently predictive of mortality. To the contrary, in a larger observational study [23] comprising outpatient HFpEF recruits and in the TOPCAT clinical trial [24], RVD did not adversely impact upon prognosis. The likely explanation for these differences include: variable HFpEF LVEF cut-offs, use of different parameters to define RVD as described earlier and more stringent exclusion criteria in clinical trials compared to community settings such as renal dysfunction or coronary artery disease which have been shown to be associated with RVD [4] but are also independently associated with increased risk [25].

Our work however adds to findings from the only 2 CMRbased HFpEF outcome studies to date $[8,9]$ and clearly implicates RVD as an important mediator of outcomes in HFpEF. In the first study [9], all surrogates of RVD, irrespective of modality (CMR, echocardiography and invasive right heart catheterization) were associated with death and or HF hospitalization during univariable analysis. In the above study ( $\mathrm{n}=142$, median follow-up 10 months), a much lower CMR measured RVEF cut-off $(<35 \%)$ was used to define RVD in contrast to our study. However, this association with outcomes was not significant for CMR RVD during multivariable analysis, following adjustment for clinical variables. 
On the other hand echocardiographic (RV systolic function, estimated pulmonary artery systolic pressure [PASP]) and invasive (measured PASP, pulmonary capillary wedge pressure [PCWP]) metrics of RVD remained independent predictors of adverse outcomes. In the second study ( $n=171$, median follow-up 573 days), RVD measured by CMR outperformed echocardiographic-derived measures of RVD as a prognostic marker [8]. The RVEF cut-off to define RVD $(<45 \%)$ was also chosen based upon ROC analysis to detect end-points. In contrast to both of the aforementioned studies, our follow-up times were substantially longer, the mere presence of RVD and not just more severe RVD was significantly associated with worse outcomes in our cohort. Furthermore, our definition of RVD was based upon reference control data, again lacking in both of these prior studies.

In line with previous studies, the RVD sub-group in our HFpEF cohort was also noted to have lower systolic blood pressure, more frequent $\mathrm{AF}[26,27]$, higher frequency of prior HF hospitalizations [21], a greater of adverse RV remodeling (RV enlargement) [27] and more prevalent pulmonary congestion [28]. There are likely multiple reasons for these findings which appear intimately linked. RV contractile function is intrinsically related to RV volumes, as also demonstrated by the moderate to strong inverse correlations of RVEF with RV volumes in our study. Furthermore, increasing RV size is an independent predictor of incident RVD development in HFpEF [27], analogous to that observed in similar LV pressure overloaded conditions such as aortic stenosis [29]. These factors either in isolation or when coupled together are known to be associated with increased venous congestion [28] as also shown by the higher rates of congestive chest radiographic changes in our RVD subjects. Increasing congestion is a major cause of HF hospitalization and therefore likely explains the observation of both prior HF hospitalization as well the association with re-hospitalization with HF (as a part of the composite end-point) seen in our RVD sub-group. Both the RVD subjects from our cohort and from previous studies $[8,9]$ also demonstrate an association with increased LA size, a surrogate marker of high LA pressure, which likely portends congestion. Furthermore, the RVD group also had a greater proportion of $\mathrm{AF}$, which is known to further exacerbate $\mathrm{RV}$ contractile dysfunction $[22,26,27]$ and provoke circulatory collapse [20] likely necessitating HF hospitalizations [30]. Our study is also the first to demonstrate an association between CMR RVEF and LAEF which likely further compounds the above features and has been hypothesized previously from echocardiographic data [22].

Other authors have previously suggested a clear relationship between RVD and the severity of left heart disease as reflected by NYHA class, natriuretic peptides or LV systolic function [5]. However, in our study, these parameters were not different between those with and without RVD. This may merely be a reflection of our sample size. Alternatively, RVD may be part of the aetiological profile in HFpEF whereby biventricular remodeling often co-exists, even in early stages [31] or driven by diffuse fibrosis secondary to systemic inflammation affecting both ventricles [32]. The correlations, albeit weak observed between RVEF and LVEF/ left ventricular end-diastolic volume indexed (LVEDVI) in our study may also be explained by a degree of ventricular interdependence driven by obesity, typical of HFpEF populations [27].

Although the observational nature of our study precludes determination of causation, AF was significantly associated with RVD, suggesting a contributory role. Our findings of a higher AF prevalence are consistent with similar reports from previous HFpEF studies [8, 21, 22, 24]. However, it remains unclear whether $\mathrm{AF}$ is a cause or consequence of RVD in HF [20]. In HFrEF, RVD reportedly predicts future AF development [33]. Irrespective of HF subtype or aetiology, AF in the setting of RVD is associated with haemodynamic instability and with poorer outcomes [20, 34]. In the HFpEF population at large, development of AF confers a poorer quality of life [30], increases hospitalization rates and worsens mortality $[30,35]$.

\section{Potential implications of our study}

Our results, through the gold standard medium of CMR reinforce previous data that RVD is present in a significant minority of HFpEF. Furthermore, the presence of RVD alone and not necessarily more severe RVD is associated with heightened risk in HFpEF. RVD may drive recurrent HF hospitalizations and mortality. Identifying RVD is potentially important for multiple reasons. HF hospitalizations are associated with significant morbidity and are a drain on healthcare resources [36]. Importantly, the prevalence of $\mathrm{HFpEF}$ is rising [3]. Understanding the mechanistic triggers for decompensation in HFpEF may also enable targeted therapies (e.g. RV focused, management of AF). Whilst treatments in unselected HFpEF patients have been neutral at best [37], one small study addressing pulmonary hypertension and RVD using a phosphodiesterase-5 inhibitor showed significant improvements in both cardiac haemodynamics and RV function [38].

\section{Strengths and limitations}

Our study is one of the largest to date evaluating RV performance utilizing CMR and also benefits from having the longest follow-up to gauge the impact of RVD on clinical outcomes. Furthermore, we also have a comparator control group which is a particular strength. While RVEF measurement is reportedly more reproducible using axial slice orientations [39], we deliberately assessed RV function from the 
short axis orientation since this is the method used routinely in clinical practice and our normative data were also derived using the same methodology [16]. Importantly, our technique yielded excellent intra- and inter-observer agreements.

This is a single centre, observational study and therefore should be replicated in additional cohorts. The association between RVD and outcome does not imply causality. We also do not have outcome data for deaths categorized as cardiovascular versus non-cardiovascular. Our definition of HFpEF was not in accordance with latest European Society of Cardiology guidelines [40]. In particular diastolic dysfunction nor elevated natriuretic peptide levels were required for diagnosis. However, diastolic dysfunction at rest is reportedly absent in nearly one-third of contemporary HFpEF clinical trials [41] and conversely also identified in a significant proportion of asymptomatic elderly subjects [41]. Only a small minority of HFpEF patients in our study had BNP levels below ESC diagnostic thresholds (14\%) which is unsurprising given the high burden of obesity observed [42]. During screening, all of our HFpEF patients subsequently enrolled had already had a diagnostic label of HFpEF made by Consultant Cardiologists during prior outpatient clinics or following a HF hospitalization. Our control group included some hypertensive subjects and was therefore not totally devoid of cardiovascular disease. Since we excluded severe lung disease (which can cause RVD), our reported prevalence of RVD is probably lower than in the general HFpEF population at large. We did not calculate (derive) estimates of pulmonary artery pressures (PAP) or quantify tricuspid regurgitation severity using echocardiography or directly assess PAP using right heart catheterization.

\section{Conclusions}

RVD as assessed by CMR is present in a significant proportion of HFpEF and is independently associated with death and or HF hospitalizations.

Acknowledgements The authors would like to thank the CMR radiographers at Glenfield Hospital for image acquisition. This work was supported by the National Institute for Health Research (NIHR) Leicester Cardiovascular Biomedical Research Centre overall project Grant: IRS_BRU_0211_20033 and the John and Lucille Van Geest Foundation. Professor GPM was supported by NIHR Research Fellowships (PDF-2011-0451 and CDF 2014-07-045). The authors would like to thank the CMR radiographers at Glenfield Hospital for image acquisition.

Author contributions PK recruited the patients, supervised the study visits and CMR scans (with AS and JNK), analyzed the data, performed the statistical analysis and drafted the initial manuscript along with JRA. PK and GPM undertook qualitative LGE image analysis. GGS undertook follow-up outcome data collection. JRA and PK performed inter-observer assessments. BNP and other serum sampling were undertaken in the hospital pathology laboratory under the supervision of PG. PK, IBS, LLN and GPM conceived the study. All authors critically revised the manuscript for important intellectual content, approved the final version for submission and agree to be accountable for all aspects of the work in ensuring that questions relating to the accuracy or integrity of any part of the work are appropriately investigated and resolved.

\section{Compliance with Ethical Standards}

Conflict of interest All authors declare that they have no competing interests relevant to this study. All authors also state that they have full control of all primary data and that they agree to allow the journal to review their data if requested.

Ethical standards The study was approved by the United Kingdom National Research Ethics Service Committee East Midlands - Nottingham (reference: 12/EM/0222). Informed consent was obtained from all individual participants included in the study. The study was performed in accordance with the ethical standards laid down in the 1964 Declaration of Helsinki and its later amendments.

Open Access This article is licensed under a Creative Commons Attribution 4.0 International License, which permits use, sharing, adaptation, distribution and reproduction in any medium or format, as long as you give appropriate credit to the original author(s) and the source, provide a link to the Creative Commons licence, and indicate if changes were made. The images or other third party material in this article are included in the article's Creative Commons licence, unless indicated otherwise in a credit line to the material. If material is not included in the article's Creative Commons licence and your intended use is not permitted by statutory regulation or exceeds the permitted use, you will need to obtain permission directly from the copyright holder. To view a copy of this licence, visit http://creativecommons.org/licenses/by/4.0/.

\section{References}

1. Di Salvo TG, Mathier M, Semigran MJ et al (1995) Preserved right ventricular ejection fraction predicts exercise capacity and survival in advanced heart failure. J Am Coll Cardiol 25(5):1143-1153

2. Gulati A, Ismail TF, Jabbour A et al (2013) The prevalence and prognostic significance of right ventricular systolic dysfunction in nonischemic dilated cardiomyopathy. Circulation 128(15):16231633. https://doi.org/10.1161/CIRCULATIONAHA.113.002518

3. Owan TE, Hodge DO, Herges RM et al (2006) Trends in prevalence and outcome of heart failure with preserved ejection fraction. N Engl J Med 355(3):251-259. https://doi.org/10.1056/ NEJMoa052256

4. Gorter TM, van Veldhuisen DJ, Bauersachs J et al (2017) Right heart dysfunction and failure in heart failure with preserved ejection fraction: mechanisms and management. Position statement on behalf of the Heart Failure Association of the European Society of Cardiology. Eur J Heart Failure 10:20. https://doi.org/10.1002/ ejhf.1029

5. Zakeri R, Mohammed SF (2015) Epidemiology of right ventricular dysfunction in heart failure with preserved ejection fraction. Curr Heart Fail Rep 12(5):295-301. https://doi.org/10.1007/s1189 7-015-0267-3

6. Champion HC, Michelakis ED, Hassoun PM (2009) Comprehensive invasive and noninvasive approach to the right ventricle-pulmonary circulation unit: state of the art and clinical and 
research implications. Circulation 120(11):992-1007. https://doi. org/10.1161/CIRCULATIONAHA.106.674028

7. Grothues F, Moon JC, Bellenger NG et al (2004) Interstudy reproducibility of right ventricular volumes, function, and mass with cardiovascular magnetic resonance. Am Heart J 147(2):218-223. https://doi.org/10.1016/j.ahj.2003.10.005

8. Aschauer S, Kammerlander AA, Zotter-Tufaro C et al (2016) The right heart in heart failure with preserved ejection fraction: insights from cardiac magnetic resonance imaging and invasive haemodynamics. Eur J Heart Fail 18(1):71-80. https://doi. org/10.1002/ejhf.418

9. Goliasch G, Zotter-Tufaro C, Aschauer S et al (2015) Outcome in heart failure with preserved ejection fraction: the role of myocardial structure and right ventricular performance. PLoS ONE 10(7):e0134479. https://doi.org/10.1371/journal.pone.0134479

10. Kanagala P, Arnold JR, Singh A et al (2020) Characterizing heart failure with preserved and reduced ejection fraction: an imaging and plasma biomarker approach. PLoS ONE 15(4):e0232280. https://doi.org/10.1371/journal.pone.0232280

11. Kanagala P, Arnold JR, Cheng ASH et al (2019) Left atrial ejection fraction and outcomes in heart failure with preserved ejection fraction. Int J Cardiovasc Imaging. https://doi.org/10.1007/s1055 4-019-01684-9

12. Kanagala P, Cheng ASH, Singh A et al (2018) Diagnostic and prognostic utility of cardiovascular magnetic resonance imaging in heart failure with preserved ejection fraction: implications for clinical trials. J Cardiovasc Magn Reson 20(1):4. https://doi. org/10.1186/s12968-017-0424-9

13. Palau P, Dominguez E, Nunez E et al (2016) Six-minute walk test in moderate to severe heart failure with preserved ejection fraction: useful for functional capacity assessment? Int J Cardiol 203:800-802. https://doi.org/10.1016/j.ijcard.2015.11.074

14. Kanagala P, Arnold JR, Singh A et al (2020) Intra-study and intertechnique validation of cardiovascular magnetic resonance imaging derived left atrial ejection fraction as a prognostic biomarker in heart failure with preserved ejection fraction. Int $\mathrm{J}$ Cardiovasc Imaging 36(5):921-928. https://doi.org/10.1007/s10554-02001785-w

15. Kanagala P, Cheng ASH, Singh A et al (2019) Relationship between focal and diffuse fibrosis assessed by CMR and clinical outcomes in heart failure with preserved ejection fraction. JACC Cardiovasc Imaging. https://doi.org/10.1016/j.jcmg.2018.11.031

16. Hudsmith LE, Petersen SE, Francis JM et al (2005) Normal human left and right ventricular and left atrial dimensions using steady state free precession magnetic resonance imaging. J Cardiovasc Magn R 7(5):775-782. https://doi.org/10.1080/109766405002955 16

17. Schulz-Menger J, Bluemke DA, Bremerich J et al (2013) Standardized image interpretation and post processing in cardiovascular magnetic resonance: Society for Cardiovascular Magnetic Resonance (SCMR) board of trustees task force on standardized post processing. J Cardiovasc Magn Reson 15:35. https://doi. org/10.1186/1532-429X-15-35

18. Singh A, Horsfield MA, Bekele S et al (2015) Myocardial T1 and extracellular volume fraction measurement in asymptomatic patients with aortic stenosis: reproducibility and comparison with age-matched controls. Eur Heart J Cardiovasc Imaging. https:// doi.org/10.1093/ehjci/jev007

19. Marcus FI, McKenna WJ, Sherrill D et al (2010) Diagnosis of arrhythmogenic right ventricular cardiomyopathy/dysplasia: proposed modification of the Task Force Criteria. Eur Heart J 31(7):806-814. https://doi.org/10.1093/eurheartj/ehq025

20. Haddad F, Doyle R, Murphy DJ et al (2008) Right ventricular function in cardiovascular disease, part II: pathophysiology, clinical importance, and management of right ventricular failure.
Circulation 117(13):1717-1731. https://doi.org/10.1161/CIRCU LATIONAHA.107.653584

21. Mohammed SF, Hussain I, AbouEzzeddine OF et al (2014) Right ventricular function in heart failure with preserved ejection fraction: a community-based study. Circulation 130(25):2310-2320. https://doi.org/10.1161/CIRCULATIONAHA.113.008461

22. Melenovsky V, Hwang SJ, Lin G et al (2014) Right heart dysfunction in heart failure with preserved ejection fraction. Eur Heart $\mathbf{J}$ 35(48):3452-3462. https://doi.org/10.1093/eurheartj/ehu193

23. Burke MA, Katz DH, Beussink L et al (2013) Prognostic importance of pathophysiologic markers in patients with heart failure and preserved ejection fraction. Circ Heart Fail. https://doi. org/10.1161/CIRCHEARTFAILURE.113.000854

24. Shah AM, Claggett B, Sweitzer NK et al (2014) Cardiac structure and function and prognosis in heart failure with preserved ejection fraction: findings from the echocardiographic study of the Treatment of Preserved Cardiac Function Heart Failure with an Aldosterone Antagonist (TOPCAT) Trial. Circ Heart Fail 7(5):740-751. https://doi.org/10.1161/CIRCHEARTFAILURE.114.001583

25. Lam CS, Donal E, Kraigher-Krainer E et al (2011) Epidemiology and clinical course of heart failure with preserved ejection fraction. Eur J Heart Fail 13(1):18-28. https://doi.org/10.1093/eurjh $\mathrm{f} / \mathrm{hfq} 121$

26. Gorter TM, van Melle JP, Rienstra M et al (2018) Right heart dysfunction in heart failure with preserved ejection fraction: the impact of atrial fibrillation. J Cardiac Fail 24(3):177-185. https ://doi.org/10.1016/j.cardfail.2017.11.005

27. Obokata M, Reddy YNV, Melenovsky V et al (2019) Deterioration in right ventricular structure and function over time in patients with heart failure and preserved ejection fraction. Eur Heart $\mathbf{J}$ 40(8):689-697. https://doi.org/10.1093/eurheartj/ehy809

28. Testani JM, Khera AV, St John Sutton MG et al (2010) Effect of right ventricular function and venous congestion on cardiorenal interactions during the treatment of decompensated heart failure. Am J Cardiol 105(4):511-516. https://doi.org/10.1016/j.amjca rd.2009.10.020

29. Genereux P, Pibarot P, Redfors B et al (2017) Staging classification of aortic stenosis based on the extent of cardiac damage. Eur Heart J 38(45):3351-3358. https://doi.org/10.1093/eurheartj/ ehx381

30. Fung JW, Sanderson JE, Yip GW et al (2007) Impact of atrial fibrillation in heart failure with normal ejection fraction: a clinical and echocardiographic study. J Cardiac Fail 13(8):649-655. https ://doi.org/10.1016/j.cardfail.2007.04.014

31. Borlaug BA, Kane GC, Melenovsky V et al (2016) Abnormal right ventricular-pulmonary artery coupling with exercise in heart failure with preserved ejection fraction. Eur Heart J 37(43):32933302. https://doi.org/10.1093/eurheartj/ehw241

32. Patel RB, Li E, Benefield BC et al (2020) Diffuse right ventricular fibrosis in heart failure with preserved ejection fraction and pulmonary hypertension. ESC Heart Fail 7(1):253-263. https://doi. org/10.1002/ehf2.12565

33. Aziz EF, Kukin M, Javed F et al (2010) Right ventricular dysfunction is a strong predictor of developing atrial fibrillation in acutely decompensated heart failure patients, ACAP-HF data analysis. J Cardiac Fail 16(10):827-834. https://doi.org/10.1016/j.cardf ail.2010.05.004

34. Tongers J, Schwerdtfeger B, Klein G et al (2007) Incidence and clinical relevance of supraventricular tachyarrhythmias in pulmonary hypertension. Am Heart J 153(1):127-132. https://doi. org/10.1016/j.ahj.2006.09.008

35. Zakeri R, Chamberlain AM, Roger VL et al (2013) Temporal relationship and prognostic significance of atrial fibrillation in heart failure patients with preserved ejection fraction: a community-based study. Circulation 128(10):1085-1093. https://doi. org/10.1161/CIRCULATIONAHA.113.001475 
36. Stewart S, Jenkins A, Buchan S et al (2002) The current cost of heart failure to the National Health Service in the UK. Eur J Heart Fail 4(3):361-371

37. Paulus WJ, van Ballegoij JJ (2010) Treatment of heart failure with normal ejection fraction: an inconvenient truth! J Am Coll Cardiol 55(6):526-537. https://doi.org/10.1016/j.jacc.2009.06.067

38. Guazzi M, Vicenzi M, Arena R et al (2011) Pulmonary hypertension in heart failure with preserved ejection fraction: a target of phosphodiesterase-5 inhibition in a 1-year study. Circulation 124(2):164-174. https://doi.org/10.1161/CIRCULATIO NAHA.110.983866

39. Alfakih K, Plein S, Bloomer T et al (2003) Comparison of right ventricular volume measurements between axial and short axis orientation using steady-state free precession magnetic resonance imaging. J Magn Reson Imaging 18(1):25-32. https://doi. org/10.1002/jmri.10329

40. Ponikowski P, Voors AA, Anker SD et al (2016) 2016 ESC Guidelines for the diagnosis and treatment of acute and chronic heart failure: the Task Force for the diagnosis and treatment of acute and chronic heart failure of the European Society of Cardiology
(ESC) developed with the special contribution of the Heart Failure Association (HFA) of the ESC. Eur Heart J 37(27):2129-2200. https://doi.org/10.1093/eurheartj/ehw128

41. Shah AM, Shah SJ, Anand IS et al (2013) Cardiac structure and function in heart failure with preserved ejection fraction: baseline findings from the echocardiographic study of the treatment of preserved cardiac function heart failure with an aldosterone antagonist trial. Circ Heart Fail. https://doi.org/10.1161/CIRCH EARTFAILURE.113.000887

42. Mehra MR, Uber PA, Park MH et al (2004) Obesity and suppressed B-type natriuretic peptide levels in heart failure. J Am Coll Cardiol 43(9):1590-1595. https://doi.org/10.1016/j. jacc.2003.10.066

Publisher's Note Springer Nature remains neutral with regard to jurisdictional claims in published maps and institutional affiliations. 